\title{
FULL WAVEFORM ACTIVE HYPERSPECTRAL LIDAR
}

\author{
T. Hakala ${ }^{\mathrm{a}, *}$, J. Suomalainen ${ }^{\mathrm{a}}$, S. Kaasalainen ${ }^{\mathrm{a}}$ \\ ${ }^{a}$ Department of Photogrammetry and Remote Sensing, Finnish Geodetic Institute, Geodeetinrinne 2, Masala, 02431, \\ Finland - (teemu.hakala, juha.suomalainen, sanna.kaasalainen)@ fgi.fi
}

KEY WORDS: Full waveform, Hyperspectral, LiDAR, Supercontinuum, Reflectance, Spectral indices

\begin{abstract}
:
We have developed a prototype full waveform hyperspectral LiDAR and investigated its potential for remote sensing applications. Traditionally hyperspectral remote sensing is based on passive measurement of sunlit targets. These methods are sensitive to errors in illumination conditions and lack the range information. Our prototype can measure both the range and the spectral information from a single laser pulse. At this stage, the instrument is optimized for short range terrestrial applications. An active hyperspectral LiDAR opens up new possibilities for LiDAR data analysis. The lack of spectral information in traditional monochrome LiDARs rules out many of the classification techniques available for processing of hyperspectral data. Similarly, passive hyperspectral data does not allow extensive use of the classifications based on 3D shape parameters. With both hyperspectral and range data available in a single dataset, the best of the techniques can be applied to form more reliable classification results. The data also allows the mapping of spectral indices in 3D. As an example a Norway spruce is measured and spatial distribution of several spectral indices is illustrated.
\end{abstract}

\section{INTRODUCTION}

Supercontinuum laser sources produce directional broadband laser pulses by making use of cascaded nonlinear optical interactions in a nonlinear optical fiber (see Dudley et al., 2006 for a review). The commercial availability of supercontinuum laser technology has lead into a number of applications in the recent years, such as those in biomedical optics (e.g., Kudlinski et al., 2010). Combined with a hyperspectral time-of-flight sensor, the supercontinuum laser sources can be used for simultaneous measurement of distance and reflectance spectrum, which has been the basis for our recent efforts in development of the hyperspectral LiDAR.

Simultaneous topographic and spectral remote sensing has thus far been based on passive imaging spectroscopy, fused with laser scanner point clouds (Jones et al., 2010; Puttonen et al., 2011; Thomas et al., 2009). Simultaneous geometric and spectral information can also be retrieved by the means of a novel approach for photogrammetric point cloud creation from automatic multispectral image matching (Honkavaara et al., 2012). Active hyperspectral imaging applications acquire a spectral signature for every pixel in the image captured by an imaging detector, but they do not produce range information (Johnson et al., 1999; Nischan et al., 2003). Range information is included in multi-wavelength laser scanners that use separate monochromatic lasers as light sources for each wavelength (Pfennigbauer and Ullrich, 2011). For these, the wavelength channels are determined by the light sources.

The hyperspectral scanning LiDAR combines active hyperspectral imaging and laser scanning with the same instrument, with no registration problems between data sets. The hyperspectral LiDAR produces a point cloud and hyperspectral reflectance: $(x, y, z, R(\lambda))$, where $R(\lambda)$ is the backscattered reflectance $R$ as a function of the wavelength $\lambda$. The information content of the new type of data is vast and creates new prospects for improving automatic data processing and target characterization for laser scanning (LiDAR) data.
We present the design of a full waveform hyperspectral LiDAR and its first demonstrations in the remote sensing of vegetation. The concept was first studied with a scanning active hyperspectral measurement system (Suomalainen et al., 2011), where the active hyperspectral intensity data was fused with simultaneous terrestrial laser scanner measurement. This lead to the development of the scanning LiDAR instrument presented in this paper (see also Hakala et al., 2012), and enabled us to study the usage of hyperspectral 3D point clouds in target classification (Puttonen et al., 2010). To our knowledge, this is the first full waveform hyperspectral LiDAR producing spectral 3D point clouds and exploiting the supercontinuum laser technology, and one of the first environmental applications of supercontinuum lasers.

\section{FULL WAVEFORM ACTIVE HYPERSPECTRAL LIDAR}

\subsection{The Instrument}

We have assembled an optical setup (Fig. 1) for measuring the time-of-flight and return intensity of a hyperspectral laser pulse. The supercontinuum laser (NKT Photonics, SuperK) produces 1 $\mathrm{ns}$ pulses at repetition rate of $24 \mathrm{kHz}$ and average power of 100 $\mathrm{mW}$. The broadband output laser is collimated using a refracting collimator (Thorlabs, CFC-5-A). The collimated beam passes through a beam sampler, which takes a part of the beam for triggering the time-of-flight measurement. An off-axis parabolic mirror $(50.8 \mathrm{~mm}$ diameter, $152.4 \mathrm{~mm}$ effective focal length and $90^{\circ}$ off-axis angle) is used as the primary collecting optic.

The off-axis parabolic mirror is focused to a spectrograph (Specim, ImSpector V10). A 16-element avalanche photo diode (APD) array module (Pacific Silicon Sensor) is used to convert the spectrally separated light to analog voltages. The APD module has built-in transimpedance amplifiers (Analog Devices, AD8015) with a bandwidth of $240 \mathrm{MHz}$ producing an unambiguous resolution of approximately $4 \mathrm{~ns}$. 12-bit analog to 
digital converters (SP Devices, ADQ412), with $1 \mathrm{GHz}$ sampling rate, are used to digitize 8 of the 16 available spectral channels. An average of 10 pulses is saved to improve signal to noise ratio and to reduce the amount of data.

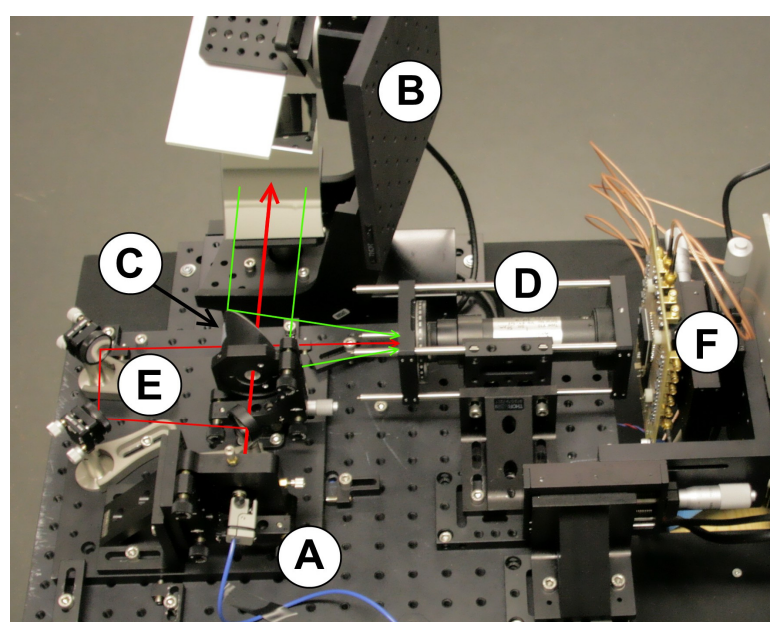

Fig.1. The optical setup: A laser pulse (A) is collimated and sent to a 2D scanner setup (B). An off-axis parabolic mirror $(C)$ is used as a primary light collecting optic. A spectrograph (D) disperses the colors of the return and trigger (E) pulses to an APD array, which converts the light to analog voltage waveforms.

The scanning geometry is defined by the two rotators (Newport URS75BCC and URS100BCC) with an absolute accuracy of $\pm 0.0115^{\circ}$. The rotators are attached to each other, with one performing the azimuth rotation and the other sweeping the laser over the target area in vertical passes. Due to uncertainty in timing, the accuracy of the elevation angles is $0.1^{\circ}$.

\subsection{Calibration and Data Processing}

A monochromator (Oriel, Cornerstone 74125) was used to calibrate the spectral responses of the APD elements. The current configuration produces spectral Full Width at Half Maximum (FWHM) of about $19 \mathrm{~nm}$ for each element and spectral range of $470-990 \mathrm{~nm}$. However, the sensitivity of the APD array and the laser intensity below $550 \mathrm{~nm}$ are low, and therefore the first channel is selected to be at $542 \mathrm{~nm}$ followed by $606,672,707,740,775,878$ and $981 \mathrm{~nm}$.

The transmitted pulse energy of the SuperK laser source may vary slightly. To take this into account, an average waveform of all spectral channels is calculated and a Gaussian peak function is fitted to the trigger part of the waveform and the waveforms are normalized with the transmit pulse intensity. Similarly, the return echo positions are detected from a mean waveform, averaged over all spectral channels. Once the return echo positions and widths are determined from the mean waveform, the hyperspectral intensities are extracted by fitting Gaussian peak heights to the spectral waveforms (Fig. 2).

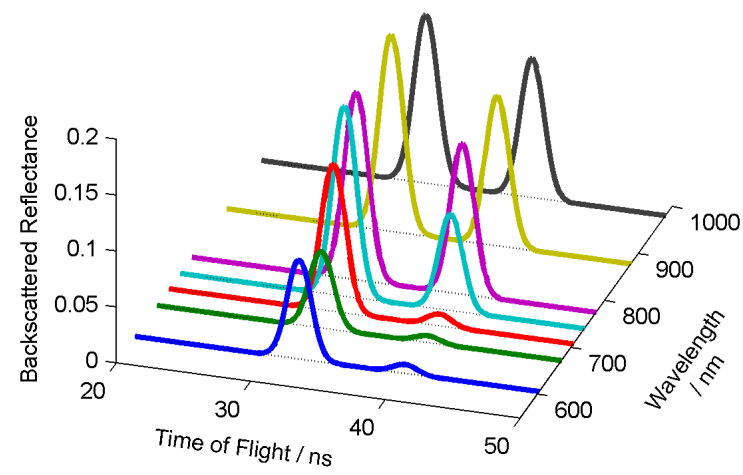

Fig. 2. Result waveforms for each channel after post processing. Gaussian peaks are fitted to each of the measured echoes. The first echo is produced by target spruce and the second by black cotton background canvas.

The intensity is converted into reflectance by applying the distance and spectral calibration. During the calibration measurements, waveforms are collected using a 99\% Spectralon as a reference target at various distances. The echo intensities are normalized with the intensity of the Spectralon echo at the same distance, producing "backscattered reflectance". As the backscattered reflectance spectra are combined with the corresponding time-of-flight and concurrent scanner orientation, a hyperspectral point cloud $(x, y, z, R(\lambda))$ is produced.

The accuracy of the measurement and the Gaussian fitting was tested by acquiring waveforms of 100 pulses reflected from a Spectralon panel at a 6-meter distance. The distance and the backscattered reflectance spectrum were retrieved individually for each pulse. The precision of ranging (standard deviation) was found to be $11.5 \mathrm{~mm}$. The precision of backscattered reflectance of a single waveform was found to be better than $2 \%$ for spectral channels within the range of 600-800 $\mathrm{nm}$ and better than $5.5 \%$ for all channels. The quality of the fit is affected by the return pulse intensity, and thus lower precision is expected for targets that are darker or further away from instrument. Higher precision can be reached by averaging over a number of measurement points. For both range and reflectance, the absolute accuracy is expected to be lower than the precision due to uncertainty in calibration.

The instrument does not have a strictly defined maximum range of measurement, as the performance of the waveform echo detection decreases slowly with the fading echo intensity. The current configuration is focused to approximately 12-meter distance. Measurements have shown that high quality point clouds can be measured from targets within 10-meter range and bright targets can be detected even from over 20 meters.

\subsection{Results and Discussion}

A Norway spruce (Picea abies) (Fig. 3) was measured in laboratory using the full waveform hyperspectral LiDAR. The bottom branches of the 2-meter spruce had suffered from lack of light and were in various stages of drying and dying, while the top branches had healthy new growth. In addition to the LiDAR measurement, reference spectra were acquired using a passive spectrometer (Avantes, AvaSpec 3648) and a quartz-tungstenhalogen light source. 


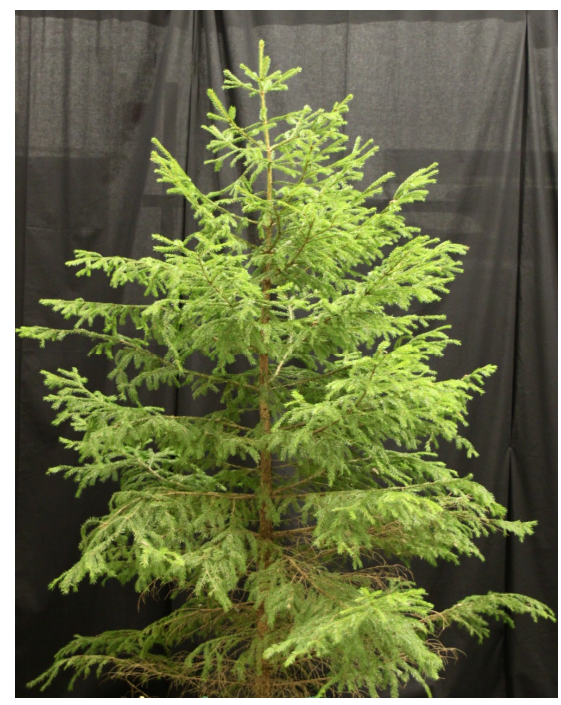

Fig. 3. The Norway spruce.

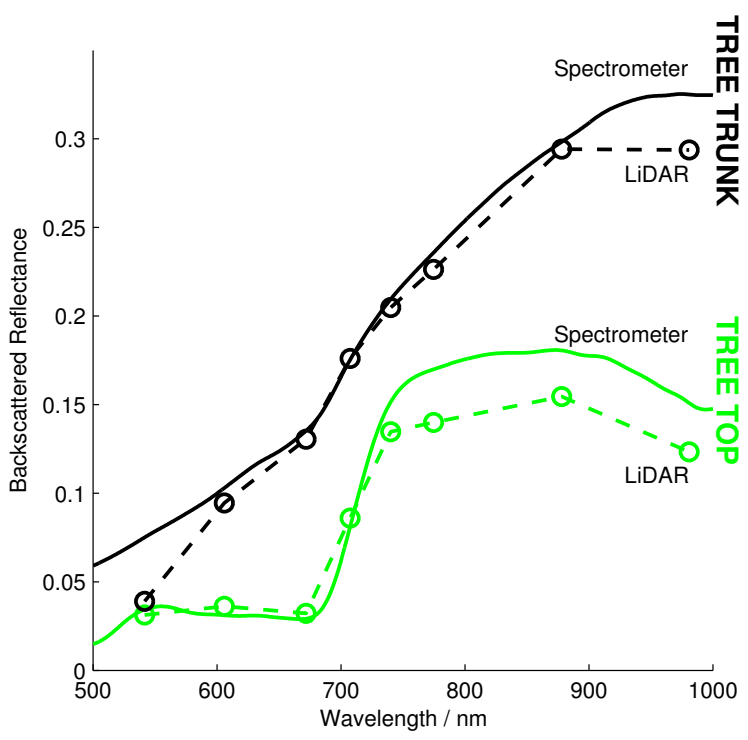

Fig. 4. Passive spectrometer measurement (solid line) and hyperspectral LiDAR (dashed line) of same areas of the tree are shown.

Spectra of the passive spectrometer and LiDAR measurements of selected regions of interest are presented in Fig. 4. A clear distinction between the tree trunk and the top can be observed in the shape of the spectra. The LiDAR and passive spectrometer spectral shapes are clearly similar. In case of the tree top, the LiDAR observes less light than the passive measurement in near-infrared. This difference is caused by multiple scattering in a medium with a low optical density and a high single scattering albedo. In an active LiDAR measurement, only a small spot on the target is illuminated and observed. A significant part of the pulse energy is lost outside the sensor field of view, if multiple scattering plays a major role in reflectance and the scattering mean free path is long in the medium. This is not experienced in passive measurement as the same amount of light is scattered both in and out of the sensor field of view. The backscattered reflectance from Spectralon is not significantly affected by this effect, as Spectralon has a high single scattering albedo but only a short mean free path. As the LiDAR backscattered reflectance is calibrated with that of the Spectralon panel, the backscattered reflectance values are decreased for bright and low optical density targets such as needles.

The backscattered reflectance values produced by the LiDAR do not strictly follow the definition of reflectance factor for three reasons: First, due to hot spot effect (Hapke, 1993), the 99\% Spectralon is not a Lambertian surface in backscattering direction causing systematic error in the reflectance values. Second, the illuminated surface area of the target is not constant (as in the definition of reflectance factor) and this results in uncertainty in the returned intensity. Third, part of the transmitted light is lost outside the sensor field of view due to multiple scattering, as described above. Despite these limitations, the backscattered reflectance is a practical quantity providing intensity readings independent of measurement distance. For most applications, the backscattered reflectance spectra can be exploited similarly to traditional reflectance factors (e.g., in the computation and comparison of spectral indices), but caution should be used when accurate absolute values are needed.

Different vegetation indices can be obtained from the measured dataset. For this study we selected Normalized Difference Vegetation Index (NDVI) (Tucker, 1979), water concentration index (Penuelas et al., 1993) and Modified Chlorophyll Absorption Ratio Index (MCARI1) (Haboudane et al., 2004). In Fig. 5, these indices have been applied to the measured dataset of the spruce.

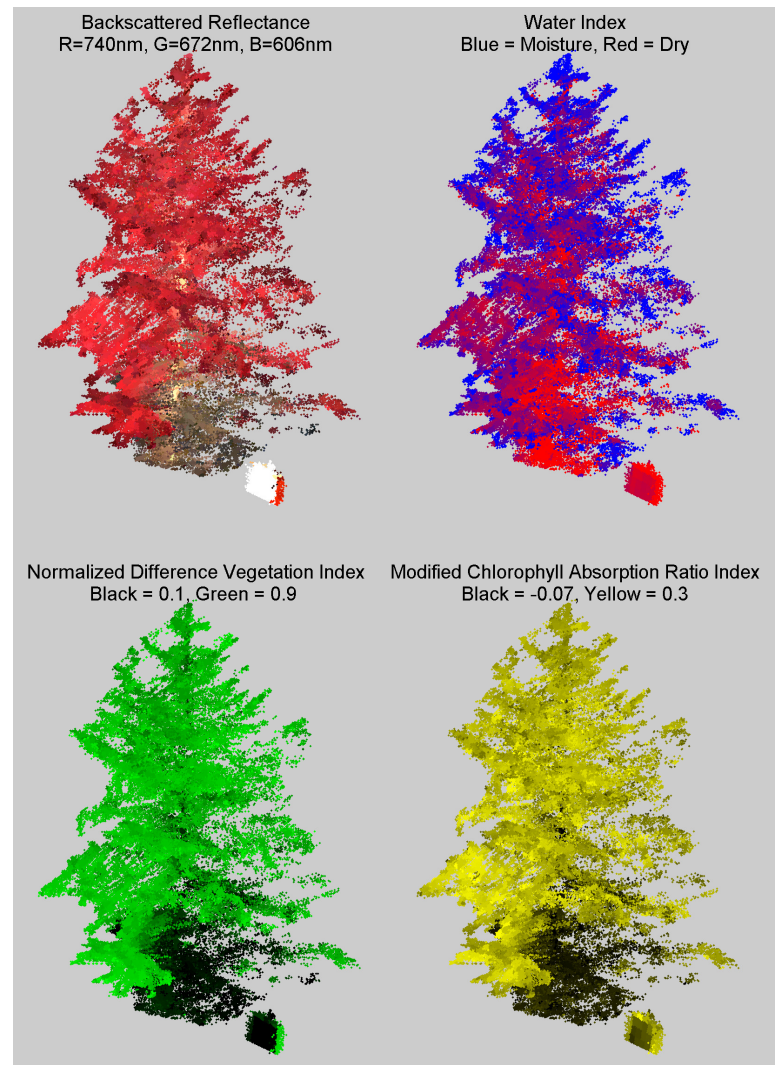

Fig. 5. Different spectral indices are calculated for $5 \mathrm{~cm}$ voxels and the full point cloud is colored according to the result. 


\section{CONCLUSION}

We present the first prototype of a full waveform hyperspectral terrestrial laser scanner and its first applications in the remote sensing of vegetation. The instrument provides a novel approach for one shot spectral imaging and laser scanning by producing hyperspectral 3D point clouds. The spectra can be used in, e.g., visualization and automated classification of the point cloud and calculation of spectral indices for extraction of target physical properties. The new type of data opens up new possibilities for more efficient and automatic retrieval of distinctive target properties, leading to improved monitoring tools for remote sensing applications, e.g., 3D-distribution of chlorophyll or water concentration in vegetation. At this stage the instrument is optimized for short range terrestrial applications, but we believe that, as technology matures, hyperspectral laser scanners with extended distance and spectral range will also become available from commercial manufacturers.

\subsection{References}

Dudley JM, Genty G and Coen S (2006) Supercontinuum generation in photonic crystal fiber. Reviews of Modern Physics 78(4): 1135.

Haboudane D, Miller JR, Pattey E, Zarco-Tejada PJ and Strachan IB (2004) Hyperspectral vegetation indices and novel algorithms for predicting green LAI of crop canopies: Modeling and validation in the context of precision agriculture. Remote Sensing of Environment 90(3): 337-352.

Hakala T, Suomalainen J, Kaasalainen S and Chen Y (2012) Full Waveform Hyperspectral LiDAR for Terrestrial Laser Scanning. Submitted to Optics Express.

Hapke B (1993) Theory of Reflectance and Emittance Spectroscopy. Cambridge, UK: Cambridge University Press.

Honkavaara E, Markelin L, Rosnell T and Nurminen K (2012) Influence of solar elevation in radiometric and geometric performance of multispectral photogrammetry. ISPRS Journal of Photogrammetry and Remote Sensing 67: 13-26.

Johnson B, Joseph R, Nischan M, Newbury A, Kerekes J, Barclay H, et al. (1999) A Compact, Active Hyperspectral Imaging System for the Detection of Concealed Targets. : International Society for Optical Engineering (SPIE).

Jones TG, Coops NC and Sharma T (2010) Assessing the utility of airborne hyperspectral and LiDAR data for species distribution mapping in the coastal Pacific Northwest, Canada. Remote Sensing of Environment 114(12): 2841-2852.

Kudlinski A, Lelek M, Barviau B, Audry L and Mussot A (2010) Efficient blue conversion from a $1064 \mathrm{~nm}$ microchip laser in long photonic crystal fiber tapers for fluorescence microscopy. Optics Express 18(16): 16640-16645.

Nischan M, Joseph R, Libby J and Kerekes J (2003) Active spectral imaging. Lincoln Laboratory Journal 14: 131-144.

Penuelas J, Filella I, Biel C, Serrano L and Save R (1993) The reflectance at the 950-970 $\mathrm{nm}$ region as an indicator of plant water status. International Journal of Remote Sensing 14(10): 1887-1905.

Pfennigbauer M and Ullrich A (2011) Multi-Wavelength Airborne Laser Scanning. : ILMF 2011.

Puttonen E, Jaakkola A, Litkey P and Hyyppä J (2011) Tree Classification with Fused Mobile Laser Scanning and Hyperspectral Data. Sensors 11(5): 5158-5182.

Puttonen E, Suomalainen J, Hakala T, Räikkönen E, Kaartinen H, Kaasalainen S, et al. (2010) Tree species classification from fused active hyperspectral reflectance and LIDAR measurements. Forest Ecology and Management 260(10): $1843-1852$.

Suomalainen J, Hakala T, Kaartinen H, Räikkönen E and Kaasalainen S (2011) Demonstration of a virtual active hyperspectral LiDAR in automated point cloud classification. ISPRS Journal of Photogrammetry and Remote Sensing.

Thomas V, McCaughey J, Treitz P, Finch D, Noland T and Rich L (2009) Spatial modelling of photosynthesis for a boreal mixedwood forest by integrating micrometeorological, lidar and hyperspectral remote sensing data. Agricultural and Forest Meteorology 149(3-4): 639-654.

Tucker CJ (1979) Red and photographic infrared linear combinations for monitoring vegetation. Remote Sensing of Environment 8(2): 127-150. 